\title{
DE L'ALTÉRATION D'HÉMOGLOBIN ET D'EAU DANS LE SANG CAUSÉE PAR LE MÉTHODE DE LA NOURRITURE DU LAPIN.
}

\author{
Par \\ HIROSHI TACHI. \\ (舘宏) \\ (Du laboratoire de physiologie du Prof. Y. Satakê de la \\ Tôhoku Université impériale de Sendai.)
}

Dans mon premier travail à propos de l'hyperglycémie hémorragique au lapin je trouvai accidentllement, au milieu de la constatation démontrant le contenu de l'hémoglobin et de l'eau du sang, son extrême irregularité dans le lapin normal. De ma statistique il résulte que le contenu d'hémoglobin va depuis 12,29 pour cent àu minimum jusquâu 22,15 pour cent àu maximum, et 17 pour cent en moyenne, et celui d'eau du sang depuis 76,94 jusqu'àu 84,56 pour cent, la moyenne ètant 81 pour cent.. ${ }^{1)}$ Puis, tout en réfléchissant, il m'est arrivé enfin de trouver que peut-être il y aurant quelque relation à une nourriture variée, qui se distinguant en son objet, serait cause du fait qu'on observe. J'en faisais l'expérience, et ètant donné le peu de travail fait dans ce champ-ci, je voulus m'assurer de la chose.

J'utilisais pour mon expérience un lapin et une lapine sains.

Dans ces expériences je n'employais que des lapins, qui avaient été ćlevés dans des niches faites en bois ou en mêtal, prouvus à l'étable d'une paille abondance, pendant une semaine au moins avant les experiences.

D'abord le système de nourriture se sépare en deux series, l'une, mettant l'animal dans une niche en métal, de la dimension de $26 \mathrm{~cm} \times 26 \mathrm{~cm}$ $\times 38 \mathrm{~cm}$, l'autre, dans une niche en bois, de la dimension de $38 \mathrm{~cm} \times 38 \mathrm{~cm}$ $\times 69 \mathrm{~cm}$, avec profusion de paille; et comme aliment, on donne $250 \mathrm{gr}$ environ de "tôfukara" tous les jours.

Pour la détermination d'hémoglobin j'employais l'hémoglobinomètre de Fleischl-Miescher, et celle de l'eau du sang, la méthode de Bang.

1) IIiro. Tach i, Tôboku J. Exp. Med., 1928, 10, 101. 
LAlteration d'IJémoglobin et l'latu dans le Sang par la Nuurriture

\begin{tabular}{|c|c|c|c|c|c|c|c|c|}
\hline & & & 泀 & 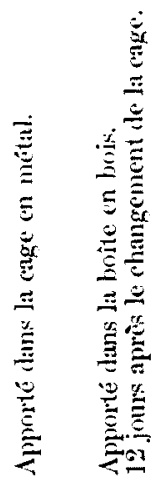 & 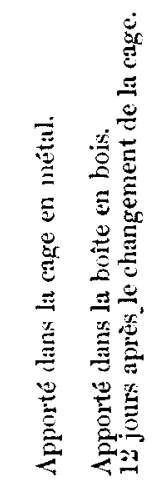 & 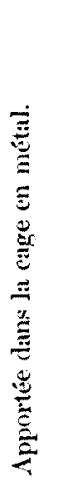 & 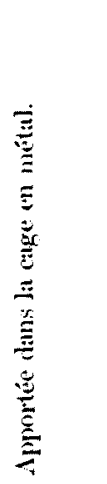 & 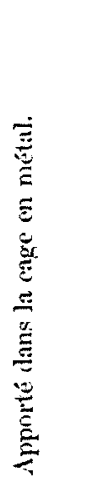 \\
\hline & axn] & & ๘ & 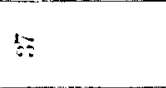 & 9 & $\stackrel{\sigma}{2}$ & $\stackrel{0}{0}$ & 67 \\
\hline & & $p$ & uвप? & 498969 & ลิ & â & aㅇ & 80 \\
\hline : & & $\mathrm{d} n$ & onanbex $\overparen{E}$ & 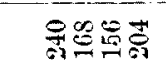 & 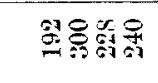 & 奂早 & 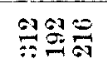 & 总䇰 \\
\hline ; & & $\begin{array}{ll}\partial \mathrm{xt} \\
\partial \boldsymbol{x}\end{array}$ & 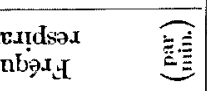 & 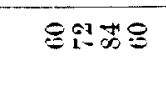 & $\Leftrightarrow$ 范落 & 89 & $\hat{1}: \cong 9$ & 草吉 \\
\hline$\Xi$ & sd $x$ & $\mathrm{np}$ & amperodurad $\underbrace{5}_{0}$ & 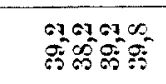 & 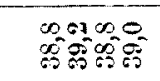 & $=0$ & 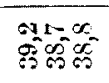 & 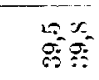 \\
\hline 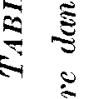 & & 0 & 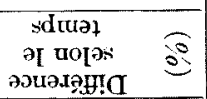 & 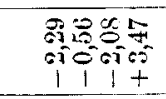 & 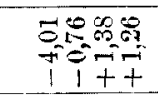 & is & $\begin{array}{l}0 \\
\text { Gig } \\
11 \\
11\end{array}$ & $\begin{array}{l}\infty 5 \\
+0 \\
10\end{array}$ \\
\hline 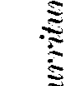 & $\varepsilon c$ & $=$ & 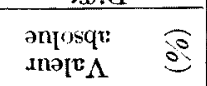 & 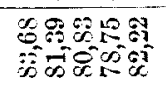 & 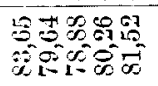 & Ho & $\begin{array}{l}950 \\
050 \\
908\end{array}$ & 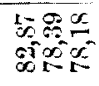 \\
\hline 2 & $\overline{\overline{\vec{H}}}$ & 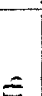 & 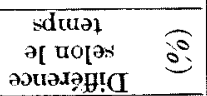 & 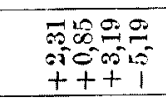 & $\begin{array}{l}3=520 \\
0=0 \\
++101\end{array}$ & $\begin{array}{c}+ \\
\text { si } \\
+\end{array}$ & $\begin{array}{l}82 \\
0= \\
+1\end{array}$ & 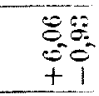 \\
\hline & & $E$ & 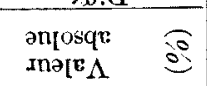 & 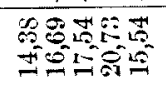 & 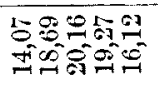 & $\begin{array}{l}695 \\
690 \\
1050\end{array}$ & 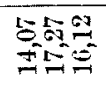 & 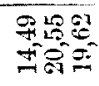 \\
\hline & 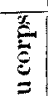 & & 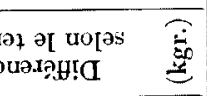 & 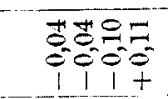 & $\begin{array}{l}61850 \\
0000 \\
+1+\end{array}$ & $\begin{array}{l}E \\
E \\
+\end{array}$ & $\begin{array}{l}3 \pm \\
0 \\
0 \\
1+\end{array}$ & $\begin{array}{l}5 \\
5 \\
0 \\
+5\end{array}$ \\
\hline & : & & squ mors & 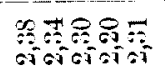 & 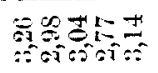 & 行 & 百果照 & 经过 \\
\hline & & & әxәs' & & $\cdots$ & cir & $c$ & to \\
\hline & & & & 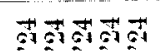 & 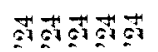 & 古它 & 实泀 & 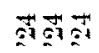 \\
\hline & & & $\stackrel{E}{E}$ & モシコシこ & ショミモう & 云 & 它ン & 急 \\
\hline & & & & 过坛家 & gojgi & ri: & $\dot{m}$ & $\dot{B \rightarrow}=$ \\
\hline & & & {$[\theta$} & - & 21 & $\therefore$ & $T$ & $\therefore=$ \\
\hline
\end{tabular}




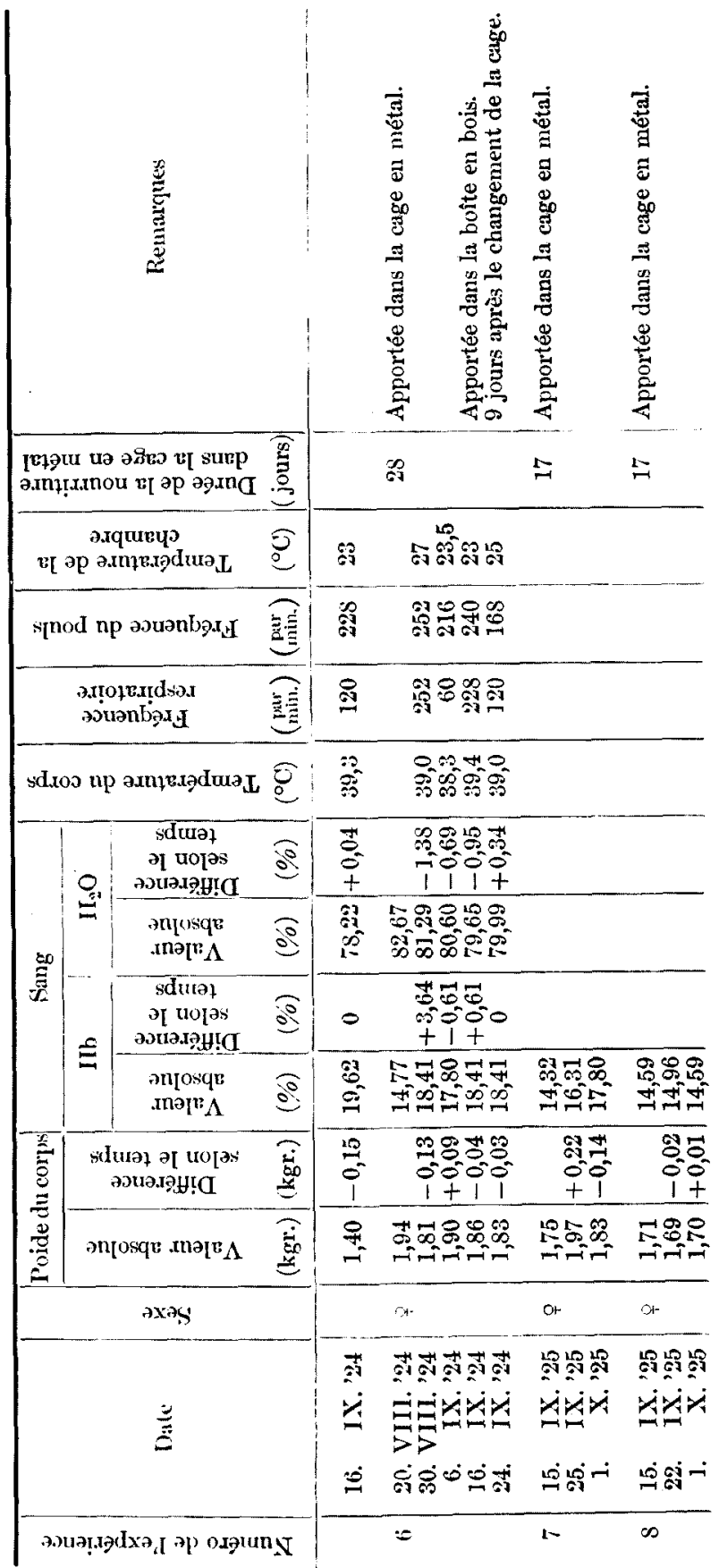

Dans la majeure partie de rette expérience dans la niche en métal, à l'exception de $\mathrm{N}^{\circ} 8$, comme le tableau premier le manifeste, les valuers d'hémoglobin montraient une augmentation, tandis que celles d'eau une diminution. Ies chiffres suivans montrent les résultats en détail : le contenu d'hémoglobin augmente de 14,49 pour cent en moyenne (14, $07-15,23 \%)$ ì 17,98 pour cent en moyenne (14, $59-20,73 \%)$; et le contenu d'ean tombe de 83,06 pour cent en moyenne $(82,54$ $-83,68 \%$ ó) ì 79 , 59 pour cent en moyenne ( 78,22 $-80,56 \%$ ); c'est$\grave{a}$-dire que ses différences ont toutes deux: quatre pour cent 


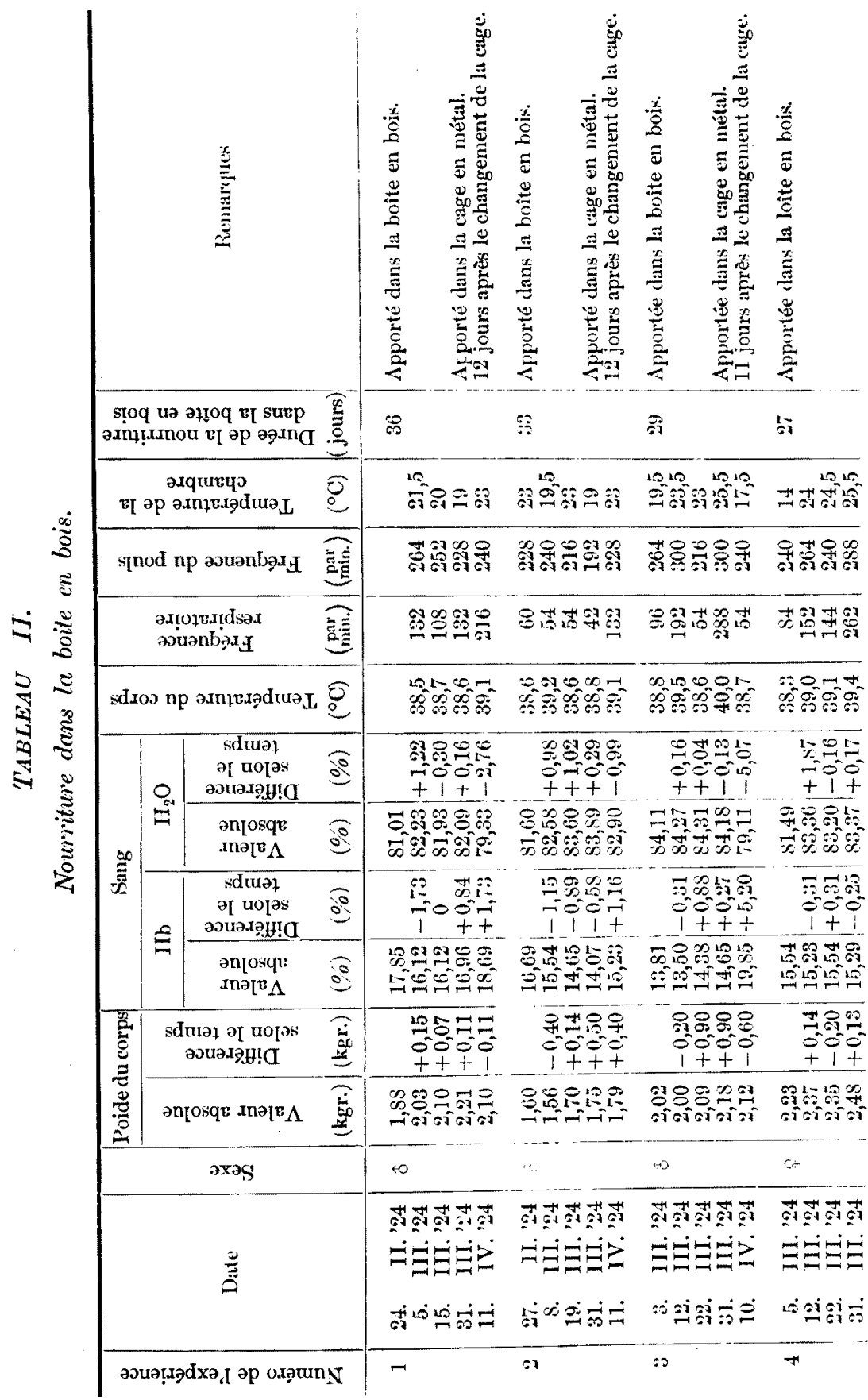




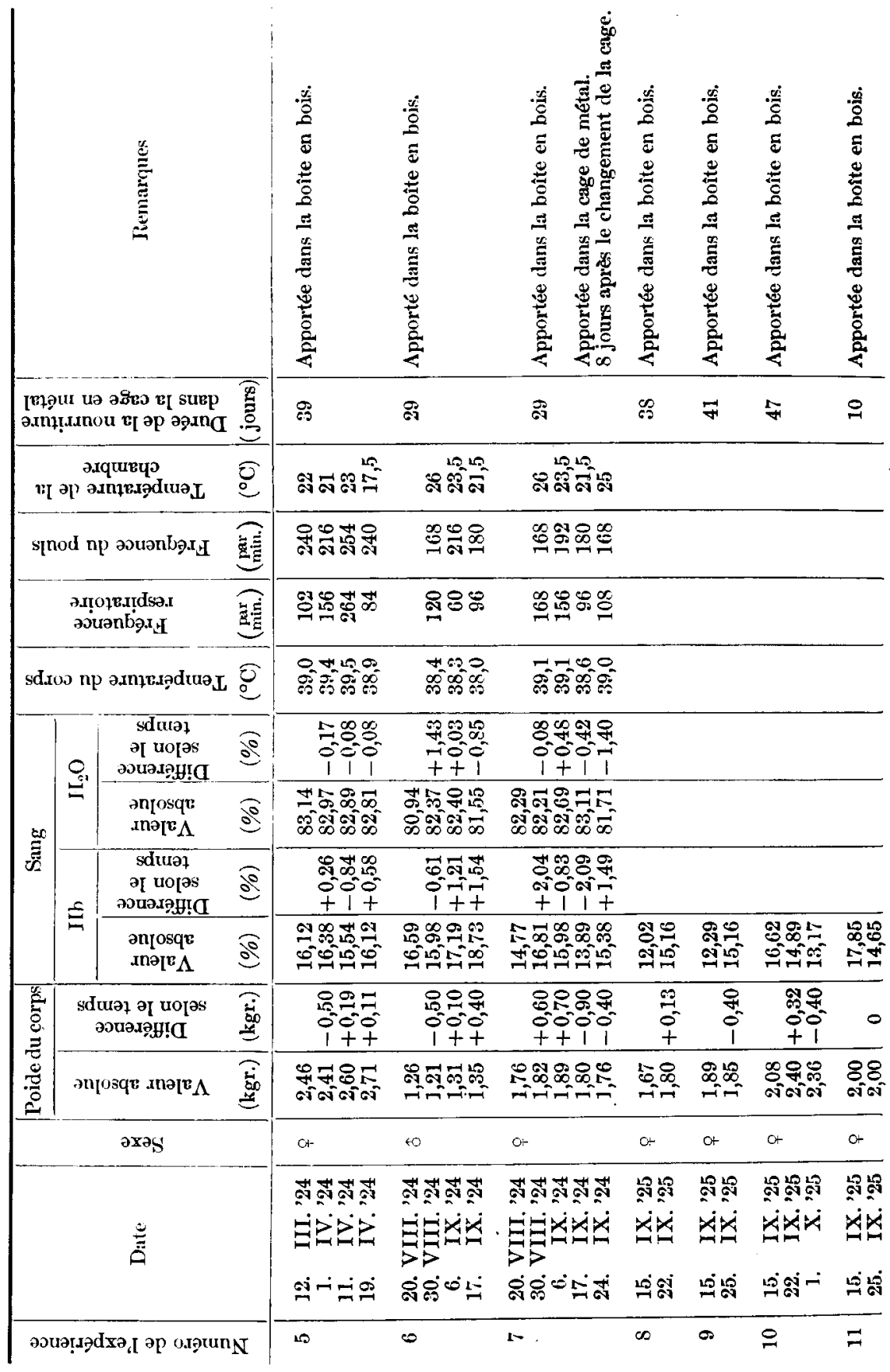


environ, dans un lapse de temps de 16 à 37 jours. Dans la niche faite en bois, le contenu d'hemoglobin change de 15,47 pour cent en moyenne $(12,02$ $-17,85 \%$ ì 15,26 pour cent en moyenne (13,17-18,73\%); et celui d'eau, de 82,08 pour cent en moyenne $(80,94-84,11 \%$ ó a 83,0 pour cent en moyenne $(81,55-84,18)$ de 10 à 38 jours c. a. d. qu'il y a peu d'altération. Dans certains cas d'expériences quoiquil y ait en moins de l'hémoglobin préalablement, ce ‘leruier manifesta plutôt de l'augmentation $\left(\mathrm{N}^{\circ} 3, \mathrm{~N}^{\circ} 8\right.$ et $N^{\circ} 9$-Tableau II).

Ce qu'il y a de plus remarquable, c'est qu'on voit que le poids du corps des animaux dans la niche de métal diminue lentement, dans la plupart des expériences, et celui des animaux dans la niche de bois, au contraire, non seulement ne diminue pas mais encore a une tendance à angmenter. Les animaux dans la niche de bois semblent avoir coutume de manger beancoup de pailles en dehors du "tôfukara." Ses fientes renfermaient les résidue de beaucoup de tissus végétaux de pailles à l'oeil nu, ct itant à l'ètat mou, tandis que celles des animaux dans la niche de métal consistaient de masses noires homogénes et dùres.

La diminution du poids du corps indique plus ou moins que le fait de l'inanition chez les animaux, expliquerait les rapports de l'hémoglobin et de l'eau dans le sang sur des animaux qui ont souffert de la faim.

Le contenu d'hémoglobin change de 15,54 pour cent en moyemne (14,$17-16,32 \%$ à à 16,98 pour cent en moyenue (15,98-18,73\%); et celui d'eau de 82,01 pour cent en moyenne $(81,28-82,98 \%)$ à 80,53 pour cent en moyenne $(79,02-81,54 \%$ ), pendant l'épreuve d'inanition de 7-15 jours.

On comprendra done que de cette expérience, une explication est donnce qui nous fait connaitre que le contenu d'hémoglobin du sang ćtait exactement en plus unrande quantité chez le lapin dans la niche de mótal que che\% celui qui était dans la niche de bois ; tandis qu'il y a, au contraire, de l'eau en moindre quantité dans celui-ci que dans celui-là. Pour résumer, je constate que jamais cet effet n'été causé seulement par l'amoindrissement du poids du corps, c. a. d. par la faim simple. Peut-être ce fait aura été influence par la faim partielle, cependant ce n'est jamais décisif. Car les altération d'hémoglobin et d'eau produités par la nourriture clans la niche de métal, sont trèssremarquables pour la baisse du poids du corps, alors que celles causées par la faim simple semblent insignifiantes et inconstantes en dépit de l'extrême amoindrissoment du poids du corps. 


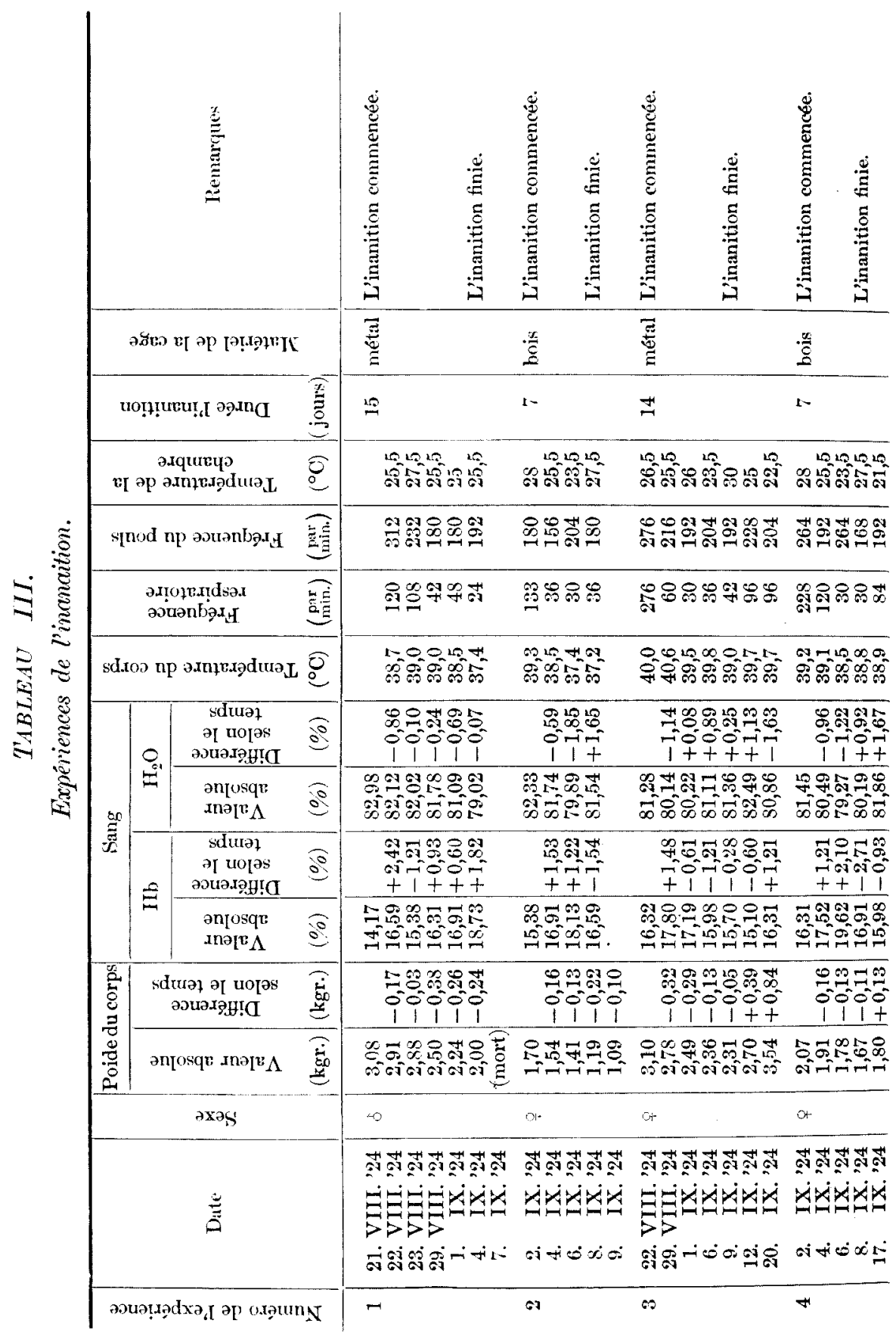




\section{Conclusion.}

Enfin, mettous donc séparément un lapin dans la niche faite en métal sans paille rien qu'avec du "tôfukara" et aussi un autre dans la niche faite en bois, avec de la grosse paille, et du " tôfukara," nous verrons que le lapin de la première niche posséde une plus grande quantite d'hémoglobin et une moindre quantité d'ean dans le sang que celui de la niche en bois. 